\title{
Comparison of Practical Implementation Limitations for Different Electronic Pre- Distortion Transmitter Structures
}

\author{
Peucheret, Christophe; Tokle, Torger; Jeppesen, Palle; Yang, Hejie
}

Published in:

European Conference on Optical Communications, 2006. ECOC 2006.

Link to article, DOI:

10.1109/ECOC.2006.4801151

Publication date:

2006

Document Version

Publisher's PDF, also known as Version of record

Link back to DTU Orbit

Citation $(A P A)$ :

Peucheret, C., Tokle, T., Jeppesen, P., \& Yang, H. (2006). Comparison of Practical Implementation Limitations for Different Electronic Pre-Distortion Transmitter Structures. In European Conference on Optical

Communications, 2006. ECOC 2006. (Vol. 3, pp. 43-44). IEEE. https://doi.org/10.1109/ECOC.2006.4801151

\section{General rights}

Copyright and moral rights for the publications made accessible in the public portal are retained by the authors and/or other copyright owners and it is a condition of accessing publications that users recognise and abide by the legal requirements associated with these rights.

- Users may download and print one copy of any publication from the public portal for the purpose of private study or research.

- You may not further distribute the material or use it for any profit-making activity or commercial gain

- You may freely distribute the URL identifying the publication in the public portal 


\title{
Comparison of Practical Implementation Limitations for Different Electronic Pre-Distortion Transmitter Structures
}

\author{
Hejie Yang, Christophe Peucheret, Torger Tokle and Palle Jeppesen \\ COM·DTU, Department of Communications, Optics \& Materials, Technical University of Denmark, \\ DK-2800 Kgs. Lyngby, Denmark. E-mail: cp@com.dtu.dk
}

\begin{abstract}
We examine different transmitter structures in electronic pre-distortion optical communication systems, and show that practical requirements due to electrical bandwidth limitation and driving signals amplitude and delay mismatches have a significant impact on system performance.
\end{abstract}

\section{Introduction}

As a major impairment in optical transmission systems, chromatic dispersion (CD) limits the system reach considerably. Traditional methods to compensate $C D$ have so far mostly focused on the optical domain. However, the use of electronic dispersion compensation (EDC) could avoid some of the limitations of the optical methods, such as insertion loss and fibre nonlinearities, and could potentially constitute a low cost solution. Consequently, EDC technologies such as feed-forward (FFE), decision feedback equalisation (DFE) and maximum-likelihood sequence estimation (MLSE) have been thoroughly investigated over the past few years [1]. Recently, a novel EDC technology using electronic pre-distortion (EPD) at the transmitter end has been proposed [2] and experimentally demonstrated [3]. Furthermore, the impact of fibre nonlinearities on EPD has also been investigated $[4,5]$. In this paper, we study three EPD transmitter structures and show that practical implementation limitations have a large effect on system performance. Numerical simulations are performed at $10 \mathrm{Gbit} / \mathrm{s}$ to compare the three modulation methods and assess their practical requirements in terms of bandwidth, driving signal amplitude mismatch and relative time delay. We show that the system performance strongly depends on the transmitter implementation and that a dual-parallel MachZehnder modulator (MZM) results in an increased tolerance to driving signals imperfections.

\section{Pre-distortion transmitter}

Given the fibre dispersion parameter $\beta_{2}$, and fibre length $z, C D$ imposes a linear operation on the electric field in the frequency domain described by $E_{R X}(\omega)=E_{T X}(\omega) \cdot \exp \left(i \beta_{2} \omega^{2} z / 2\right)$, where $E_{R X}(\omega)$ and $E_{T X}(\omega)$ are the Fourier transforms of the received and transmitted electric fields envelopes, respectively. Therefore, the field at the transmitter $E_{T X}(t)$ can be calculated from the desired received signal. The aim of the EPD transmitter is to generate the calculated $E_{T X}(t)$ so that dispersion can result in the desired signal at the output of the link.

Three proposed transmitter structures are shown in Fig. 1. In all three cases, the input binary data pattern is processed in order to generate two pre-distorted electrical signals. In Fig. 1(a), a simple MZM is used to modulate a continuous wave (CW) from two driving

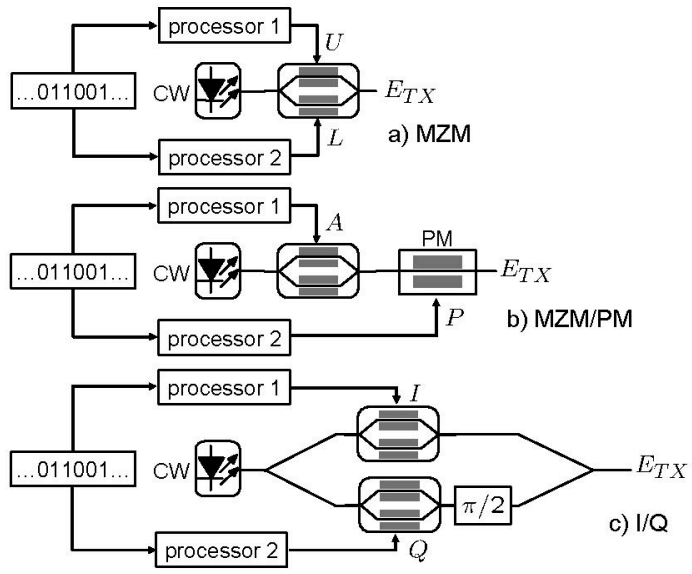

Fig. 1: Transmitter structures. a) single $M Z$ modulator $(M Z M)$; b) $M Z$ modulator and phase modulator (MZM/PM); c) dual-parallel MZ modulator (I/Q)

signals denoted $U$ and $L$. In Fig. 1(b), the desired predistorted signal is obtained from intensity modulation in a MZM driven by the signal $A$, followed by a phase modulator driven with the signal $P$. Alternatively an inphase and quadrature (I/Q) optical modulator made of a dual parallel MZM driven by the two pre-distorted electrical signals $I$ and $Q$ is also presented in Fig. 1(c). The three different transmitter structures are denoted MZM, MZM/PM and I/Q. The processors used to generate the driving signals are described for instance in [2], where a look-up table and a digital-toanalogue (D/A) converter are used. The target optical signal $E_{R X}$ is identical for the three investigated transmitter structures. However the electrical predistortion waveforms are different due to the different modulator transfer functions. Therefore, if practical requirements such as bandwidth limitation of the modulators, amplitude deviation or delay mismatch, between the driving signals are introduced, different system performances are expected.

\section{Simulation results}

Simulations are carried out to assess the performance of the proposed transmitter structures at $10 \mathrm{Gbit} / \mathrm{s}$. The input binary data pattern is a $2^{7}-1$ pseudo random bit sequence (PRBS). The transmission span consists of $200 \mathrm{~km}$ standard single mode fibre (SSMF). In the present investigation, the effects of fibre nonlinearities and noise are ignored. The receiver is a photodiode followed by a $4^{\text {th }}$ order low- 
pass Bessel filter with $7.5 \mathrm{GHz} 3 \mathrm{~dB}$ cut-off frequency. The target received signal is un-chirped NRZ with rise time equal to one tenth of the bit duration (defined in the optical domain). For both MZM/PM and I/Q transmitters, $\mathrm{X}$-cut single drive MZMs are assumed.

For a given bit slot, the pre-distorted waveform is calculated by taking into account 3 pre- and post-bits (i.e. the buffer length is set to 7 in the processor), which has been shown to be sufficient for $200 \mathrm{~km}$ SSMF transmission [2]. The sample rate of the D/A converter is chosen large enough to ensure no penalty is introduced at this stage.

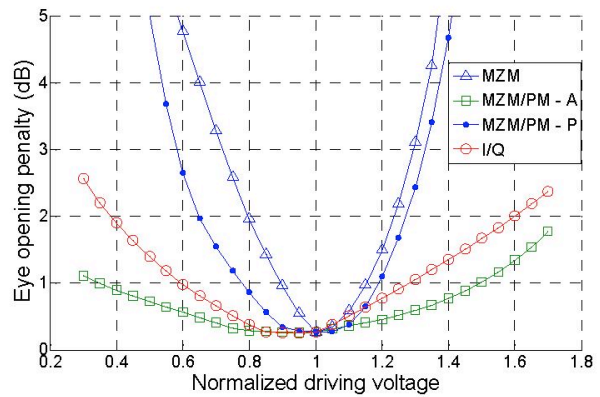

Fig. 2: EOP as a function of normalized driving voltage.

First, the effect of the driving signals amplitude deviation (compared to the ideal driving voltages) on the eye-opening penalty (EOP) is investigated. As shown in Fig. 2, the MZM is the most sensitive structure to amplitude deviation. A variation of $\pm 20 \%$ from the ideal driving voltage results in a $2 \mathrm{~dB} E O P$. The MZM/PM transmitter is fairly sensitive to amplitude fluctuations of the $P$ signal, while it is robust to amplitude variations of the $A$ signal.

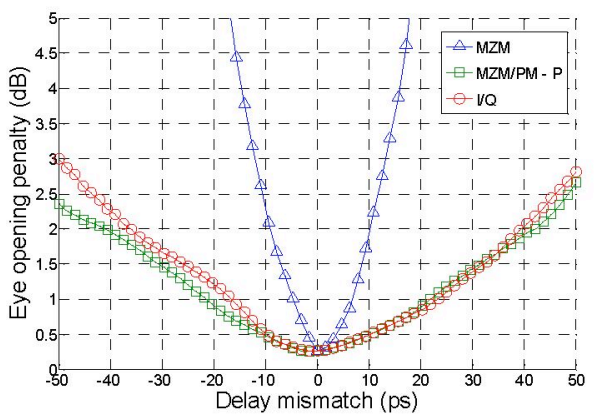

Fig. 3: EOP as a function of delay mismatch between the driving signals.

Second, the dependence of the EOP on the delay mismatch between the driving signals is investigated, as illustrated in Fig. 3. It can be seen that the single $M Z M$ is the most sensitive structure to delay mismatch, while the MZM/PM and I/Q structures have a much larger tolerance. The MZM would result in $2 \mathrm{~dB}$ EOP when the delay mismatch is beyond \pm 10 ps, while the MZM/PM or $/ / Q$ would only suffer from $0.5 \mathrm{~dB}$ EOP. Comparing Fig. 2 and Fig. 3, it can be seen that the delay mismatch is more critical than amplitude deviations. In the I/Q case, less than $1.5 \mathrm{~dB}$ penalty arises even if the amplitude deviates as much as $50 \%$ of the desired value, while \pm 30 ps delay mismatch gives the same penalty. The allowable deviations are summarised in Table 1 for a $1 \mathrm{~dB}$ penalty. It is shown that the MZM transmitter is the most sensitive to both amplitude and delay imperfection, while the requirements for the two other structures are more relaxed.

\begin{tabular}{|c|c|c|}
\hline \multirow{2}{*}{$\begin{array}{l}\text { Driving } \\
\text { signals }\end{array}$} & Normalized Amp. & Delay mismatch (ps) \\
\hline & $\min . \rightarrow \max$ & $\min . \rightarrow \max$ \\
\hline MZM & $\rightarrow 1.15$ & $-5 \rightarrow+7$ \\
\hline MZM/PM - A & $\rightarrow \quad 1.4$ & $-21 \rightarrow+21$ \\
\hline MZM/PM - P & 1.2 & $-15 \rightarrow+12$ \\
\hline $\mathrm{I} / \mathrm{Q}$ & 0.6 & $-16 \rightarrow+14$ \\
\hline
\end{tabular}

Table 1: $1 \mathrm{~dB}$ penalty tolerance of amplitude deviation and delay mismatch.

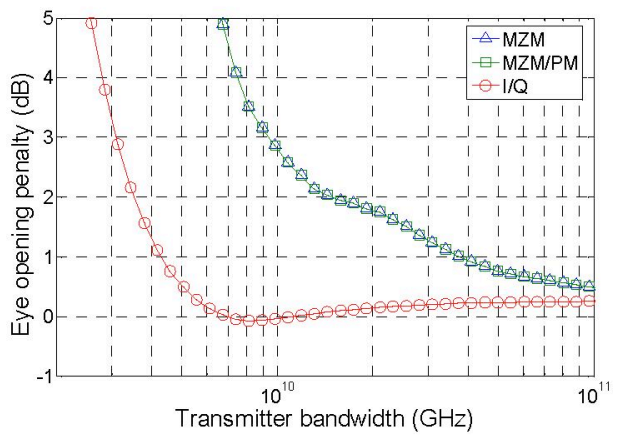

Fig. 4: EOP versus transmitter bandwidth, for single $M Z M, M Z M / P M$ and $1 / Q$ transmitters

Finally, the effect of bandwidth limitation of the MZM or PM is examined. It is implemented by filtering the driving signals using $4^{\text {th }}$ order low-pass Bessel filters. As shown in Fig. 4, the $I / Q$ structure has an advantage of more than $1 \mathrm{~dB}$ penalty when its bandwidth varies from 10 to $30 \mathrm{GHz}$. More than $1 \mathrm{~dB}$ penalty would be observed at $10 \mathrm{Gbit} / \mathrm{s}$ even if the modulator bandwidth is as large as $40 \mathrm{GHz}$ in the MZM and $M Z M / P M$ cases, clearly demonstrating the unsuitability of those structures.

\section{Conclusion}

We have compared three pre-distortion transmitter structures. A single MZM would hardly comply to practical requirements induced by bandwidth limitation, delay mismatch and amplitude deviation. Simulation results show that $I / Q$ modulators offer many more advantages as the modulation structure in predistortion transmitters for dispersion compensation.

\section{References}

1 G. Prati, ECOC'05, Mo4.6.

2 R. I. Killey et al., IEEE Photon. Technol. Lett., 17 (2005) 714-6

3 D. McGhan et al., IEEE Photon. Technol. Lett., 18 (2006) 400-2.

4 R.-J. Essiambre et al., ECOC'05, Tu3.2.2.

5 K. Roberts et al., IEEE Photon. Technol. Lett., 18 (2006) 403-5. 